Artigo de Revisão

\title{
A percepção auditiva nos pacientes em estado de coma: uma revisão bibliográfica
}

\author{
The auditory perception in the patients in state of coma: a bibliographical revision \\ La percepción auditiva en los pacientes en estado de coma: una revisión bibliográfica
}

\begin{abstract}
Ana Cláudia Giesbrecht Puggina ${ }^{1}$, Maria Júlia Paes da Silva ${ }^{2}$, Maria Fernanda Zorzi Gatti ${ }^{3}$, Kazuko Uchikawa Graziano ${ }^{4}$, Miako Kimura ${ }^{5}$
\end{abstract}

\begin{abstract}
RESUMO
Muito ainda precisa ser estudado pela neurociência, tanto em relação aos estados de consciência quanto aos processos cognitivos e a percepção auditiva nos estados de coma. Assim, esse estudo de revisão bibliográfica sobre a percepção auditiva dos pacientes em estado de coma parece ser relevante no sentido de trazer maiores esclarecimentos e identificação de evidências. Identificar, nas publicações, as lacunas, consistências e inconsistências desse tema. O estudo consiste em levantamento bibliográfico nas bases de dados LILACS e PUBMED. Os artigos foram analisados quanto ao método e os principais resultados do estudo. Foram selecionados 10 artigos sobre o tema, sendo nenhum deles nacional. A maior parte dos estudos selecionados foi publicada no período de 1971-1995 (80\%), pelos Estados Unidos (70\%), como pesquisa longitudinal $(50 \%)$ ou relato de caso (30\%); a amostra variou entre 1 e 5 (70\%), a maioria usou a música (80\%); o EEG (60\%) e a observação comportamental $(50 \%)$ foram as medidas de resposta ao estímulo mais utilizadas. Os procedimentos foram variados, porém a maioria dos estudos, apontam para a existência de uma percepção auditiva nos pacientes em estado de coma.
\end{abstract}

Descritores: Percepção auditiva; Coma

\begin{abstract}
Much is still needed to be discovered by the neuroscience in relation to the consciousness regarding cognitive processes and the auditive perception in coma. Thus, this study is a bibliographic review on the auditive perception of patients in coma and shows its relevance to explain and identify evidence. To identify, in the publications found, the gaps, consistencies and inconsistencies on this subject. This study consists of a bibliographical survey based on LILACS and PUBMED database. Ten articles were selected about the subject, none of them are national publications. Most of the selected studies were published within the period of 1971-1995 (80\%), by the United States (70\%), as longitudinal research $(50 \%)$ or study of case (30\%); the sample in general varied between 1 and $5(70 \%)$, the majority used music (80\%); the EEG (60\%) and behavior observation $(50 \%)$ were the most used measuring ways of the responses to the stimulus. The procedures were varied, however most of the studies, show that there is an auditory perception in patients with coma.
\end{abstract}

Keywords: Auditory perception; Coma

\section{RESUMEN}

Mucho aún se hace necesario a ser estudiado por la neurociencia, tanto en relación a los estados de conciencia cuanto a los procesos cognitivos y la percepción auditiva en lo estado de coma. Así, este estudio de revisión bibliográfica sobre la percepción auditiva de los pacientes en estado de coma parece ser relevante para traer mayores esclarecimientos e identificar las evidencias. Identificar, en las publicaciones encuentradas, las lacunas, las consistencias y las inconsistencias de este tema. Este estudo consistió en un levantamiento bibliográfico en las bases de datos LILACS y PUBMED. Para esto, los artículos fueron analizados de acuerdo con el método y los resultados principales del estudo. Fueron seleccionados 10 artículos sobre el tema, ninguno de ellos de la publicación nacional. La mayoría de los estudios seleccionados fueron publicados en el período de 1971-1995 (80\%), en los Estados Unidos (70\%), como la pesquisa longitudinal (el 50\%), estudo del caso (30\%); la muestra en general varió entre 1 y 5 (el 70\%), la mayoría utilizaron la música (el 80\%); el EEG (el 60\%) y el observación comportamental (50\%) habían sido las medidas de respuesta más usadas. Los procedimientos habían sido muy variados, no obstante de la mayoría de los estudios, enseñan para la existencia de una percepción auditiva en los pacientes en estado de coma. Descriptores: Percepción auditiva; Coma

${ }^{1}$ Enfermeira. Mestranda do Programa de Saúde do Adulto e do Idoso da Escola de Enfermagem, Universidade de São Paulo - USP-São Paulo (SP), Brasil. Bolsista FAPESP.

${ }^{2}$ Professora Titular do Departamento de Enfermagem Médico-Cirúrgica da Escola de Enfermagem, Universidade de São Paulo - USP - São Paulo (SP), Brasil.

${ }^{3}$ Enfermeira. Especialista em Terapia Intensiva e Pronto Socorro. Mestranda da Escola de Enfermagem, Universidade de São Paulo - USP - São Paulo (SP), Brasil. Chefe de Enfermagem do Pronto Socorro Adulto do Hospital Samaritano - SP.

${ }^{4}$ Enfermeira. Professora Livre-docente do Departamento de Enfermagem Médico-Cirúrgica da Escola de Enfermagem, Universidade de São Paulo - USP - São Paulo (SP), Brasil.

${ }^{5}$ Enfermeira. Professora Livre-docente do Departamento de Enfermagem Médico-Cirúrgica da Escola de Enfermagem, Universidade de São Paulo - USP - São Paulo (SP), Brasil.

Autor Correspondente: Ana Cláudia Giesbrecht Puggina

Rua da Consolação, 927 - Ap. 157 01301-000 São Paulo - SP

Artigo recebido em 03/01/05 e aprovado em 08/04/05

E-mail: claudiapuggina@hotmail.com 


\section{INTRODUÇÃO}

O cérebro é um órgão como outro qualquer do organismo humano, porém muito mais complexo. Com o desenvolvimento de novas tecnologias, esse órgão onde se situa nosso coração metafórico, vai perdendo a aura de mistificação e apontando novos caminhos para aumentar a qualidade de vida ${ }^{(1)}$.

Os conceitos de consciência, até hoje, enfrentam o desafio de dar conta da relação entre a experiência subjetiva e os processos físicos do cérebro ${ }^{(2)}$. James $^{(2)}$, em 1890, coloca a questão que vai estar presente em toda a Psicologia científica subseqüente, a de preencher "o abismo explicativo que se coloca entre os mundos interno e externo" ou, mais modernamente, entre uma experiência subjetiva e um cérebro físico.

O obstáculo mais difícil de se transpor na investigação científica da consciência, talvez esteja relacionado à concepção culturalmente arraigada de que percepções conscientes não podem ser consideradas como fruto do funcionamento do sistema nervoso, sendo a consciência uma entidade distinta deste, e que apenas manifestase através dessa estrutura. Parte substancial desse viés dualista deve-se a dogmas que estabelecem uma separação entre o espírito e o corpo ${ }^{(3)}$.

O fenômeno "consciência" não é uma entidade única, mas sim um conjunto de habilidades mediadas pelo processamento paralelo, porém cooperativo, de informações em diferentes módulos do sistema nervoso. Não obstante essa organização modular, o funcionamento cooperativo e integrado dos diferentes módulos produz uma sensação unificada ${ }^{(3)}$.

Igualmente não muito bem descrito encontra-se o fenômeno da inconsciência pacientes em estado de coma considerados "inconscientes" intrigam muitos pesquisadores. O coma pode ser definido como um estado em que o indivíduo não demonstra conhecimento de si próprio e do ambiente, caracterizado pela ausência ou extrema diminuição do nível de alerta comportamental, permanecendo não responsivo aos estímulos internos e externos e com os olhos fechados ${ }^{(4)}$.

A experiência do coma é um processo complexo que pode deixar profundas marcas em quem o vivenciou. Muitas destas marcas não estão somente ligadas ao coma em si, mas às experiências de ser "des/cuidado" durante esse processo, levando muitos doentes não só a necessitarem de se recuperar da doença, mas do fato de terem se tornado "pacientes". Isto porque, apesar dos avanços teóricos acerca do cuidado, a prática ainda se dá quase que, exclusivamente, com base em ações profissionais despersonalizadas, na qual o ser se torna a doença, o objeto passivo da investigação e do tratamento ${ }^{(5)}$.

A incidência de pacientes em estado de coma nas Unidades de Terapia Intensiva depende de inúmeros fatores, tanto em relação à complexidade de assistência que o hospital oferece quanto em relação ao tipo de população atendida; porém, estima-se que mais de $80 \%$ dos pacientes encontram-se nesse estado por coma farmacológico, fisiológico, metabólico etc.

O tratamento em unidades de terapia intensiva é um ramo da Medicina altamente tecnológico. Mesmo em casos aparentemente sem esperanças, pode-se salvar vidas através de aplicação de modernas tecnologias. Porém, embora esse contexto de sucesso, o tratamento em unidades de terapia intensiva tem falhado em outro aspecto. Pacientes parecem sofrer de outros problemas resultados da comunicação insuficiente, alteração no sono e falta de empatia entre paciente e equipe. Muitas atividades em situações de cuidado intensivo parecem ser entre a equipe e as máquinas, sujeitos e objetos ${ }^{(6)}$.

Durante esse estado clínico, a audição desses pacientes sempre foi muito questionada; porém os trabalhos presentes na literatura para validar essa questão são poucos e estão muito dispersos.

Nos estados de coma a audição parece ser o último sentido que é perdido e tal afirmação pode ser sustentada através dos relatos de pessoas que retornaram desse estado. A maioria relata dados sensoriais auditivos como sons, palavras, frases, vozes familiares etc.

A percepção auditiva, como os outros sentidos, passa por três etapas básicas: um estímulo físico; um conjunto de eventos pelos quais o estímulo é transduzido em uma mensagem de impulsos nervosos; uma resposta à mensagem, freqüentemente como uma percepção ou uma representação interna das sensações ${ }^{(7)}$. Recebemos o som através de ondas de pressão; esse estímulo físico é transformado em estímulo eletroquímico, entretanto como conseguimos transformar essa informação auditiva em significado, constitui o milagre da audição. Parte da nossa capacidade para imprimir coerência a tudo isso deve-se ao fato de que desenvolvemos modelos do que esperamos ouvir: fonemas, palavras, música ${ }^{(8)}$.

Em uma Unidade de Terapia Intensiva, uma atmosfera sonora extremamente particular, alarmes de bombas de infusão, monitores eletrocardiográficos e ventiladores mecânicos, envolvem o paciente e podem deixá-lo mais apreensivo. Além disso, não podemos deixar de considerar toda a comunicação existente ao lado do leito do paciente, desde num banho de leito até numa passagem de plantão, em que seu nome e seu estado clínico é claramente mencionado. Quantas vezes em uma passagem de plantão leito a leito, são passadas para a equipe de enfermagem subseqüente, informações tais como: "não iremos mais investir nesse paciente" ou "este paciente não é candidato à ressuscitação"? Por mais absurda que essa situação pareça ela acontece com grande freqüência. E quem nos garante que esses pacientes não nos podem ouvir?

Cuidar holisticamente de um paciente em coma é uma tarefa exigente, principalmente porque o estado de coma 
do outro, parece nos deixar, também em certa suspensão. A compreensão mais profunda sobre a consciência humana e sobre o papel relevante da mente nos processos de reequilíbrio vital pode contribuir, não só para minimizar a ansiedade experimentada pelos profissionais em muitos casos, mas também para estender as intervenções para além do cuidado físico (de importância irrefutável) e do controle técnico dos monitores (controle fisiológico), buscando atingir as demais dimensões humanas, potencializando-as tanto quanto possível ${ }^{(9)}$.

Muito ainda precisa ser estudado pela neurociência, tanto em relação aos estados de consciência, quanto aos processos cognitivos e a percepção auditiva nos estados de coma. Portanto, esse estudo de revisão bibliográfica sobre a percepção auditiva dos pacientes em estado de coma parece ser relevante, no sentido de trazer maiores esclarecimentos e identificação de evidências.

\section{OBJETIVO}

Analisar os estudos publicados que abordem a audição nos pacientes em estado de coma, com a finalidade de identificar, nas publicações encontradas, ano de publicação, país de origem da publicação, tipo de publicação, amostra, medidas de resposta ao estímulo auditivo aplicado, estímulos auditivos utilizados, propósitos do estudo, procedimentos e principais resultados.

\section{MÉTODOS}

Este estudo caracteriza-se como de natureza descritiva, retrospectiva, de revisão bibliográfica. O objeto de estudo deste trabalho foram os artigos publicados sobre o tema em questão, em periódicos nacionais e internacionais, indexados e especializados na área de saúde.

Os critérios de inclusão foram: artigos publicados em periódicos nacionais e internacionais que constaram nas bases de dados definidas para este estudo (LILACS e PubMed); artigos acessados na íntegra; escritos em inglês, português ou espanhol; pacientes adultos em estado de coma; artigos que referem-se ao sentido da audição nesses pacientes.

Para coleta dos dados foi elaborado um instrumento contendo os seguintes itens: identificação do artigo (título, periódico e autores); ano de publicação; país de origem da publicação; tipo de publicação (pesquisa transversal, pesquisa longitudinal, relato de caso e caso-controle); amostra; medidas de resposta ao estímulo auditivo aplicado; estímulos auditivos utilizados; procedimentos; propósitos do estudo; principais resultados.

\section{RESULTADOS}

O levantamento bibliográfico dos artigos foi realizado mediante busca eletrônica em duas bases de dados:
PubMed e LILACS, bases de dados gerais da área da saúde. O levantamento bibliográfico compreendeu todo o período disponível em cada base de dados.

$\mathrm{Na}$ base de dados PubMed, os seguintes descritores de assunto (MeSH) foram cruzados usando o recurso boleano "and" (Tabela 1):

$\mathrm{Na}$ base de dados LILACS não foi identificada nenhuma publicação cruzando os mesmos descritores de assunto (DeSC) em português.

Inicialmente, foi feita uma leitura exploratória dos 43 artigos através dos títulos e resumos para o reconhecimento dos artigos de interesse. A seguir, foi realizada uma leitura seletiva dos artigos na íntegra, no intento de escolher os que, de fato, servissem aos propósitos desse estudo. Dessas leituras foram excluídos 33 artigos (Tabela 2).

Selecionamos para análise somente aqueles que atendiam aos critérios de inclusão pela leitura do resumo ou do trabalho na íntegra. Portanto, a amostra do estudo compreende 10 artigos.

Em relação ao ano de publicação observou-se que a maior parte dos trabalhos foi publicada no período de 1971-1995 (8 dos 10), evidenciando a necessidade de mais estudos recentes sobre o tema.

Considerando o país de origem da publicação observou-se uma hegemonia norte americana, sendo que 7 dos 10 artigos foram publicados nos Estados Unidos, e uma ausência de publicação nacional sobre esse tema.

Os tipos de publicação predominantes foram a pesquisa longitudinal (5 dos 10 artigos) e o relato de caso (3 dos 10 artigos).

Quanto a amostra, na maioria dos estudos (7 dos 10) o número da amostra variou entre 1 e 5 , mostrando uma certa dificuldade na coleta de dados com esse tipo de paciente e estado clínico.

Em relação aos estímulos auditivos, a maioria dos trabalhos (8 dos 10 artigos) utilizou música. Os estímulos foram muito variados e criativos, mas com esses pacientes houve uma predominância (4 dos 10 estudos) em se optar pelo uso de músicas da preferência do paciente.

Os estímulos auditivos foram estímulo "click" em dois estudos; música da preferência do paciente (country e gospel); estímulos verbais e música de preferência do paciente depois de comportamentos almejados; 2 músicas populares e anedota; música clássica, rock'n roll (estilo de preferência), sons da natureza e vozes familiares; música clássica de piano, barulhos ambientais da unidade e gravação com redução de ruídos dos barulhos da unidade; canção melódica sem palavras cantada pela terapeuta de acordo com a preferência musical do paciente, sua pulsação e seu ritmo respiratório; música de Mozart (Sonata for Two Pianos em D major) e a música controle (Old Time Pop Piano Tunes); timbres (clarineta, harmônica etc).

Talvez o principal motivo pelo uso tão abrangente da música seja o seu poder em estabelecer contato sem a 
linguagem, através dela podemos encontrar um potencial não utilizado em outros meios de comunicação. Uma vez que a música propicia um meio de comunicação de caráter predominantemente emocional (comunicação não-verbal e pré-verbal), ela tem importância e grande aplicação, exatamente onde a comunicação verbal não é utilizada ${ }^{(10)}$.

Dentre as medidas de resposta ao estímulo auditivo aplicado, o eletroencefalograma (EEG) foi usado em 6 dos 10 estudos. Destes que utilizaram esse recurso, 4 foram realizados entre 1971 e 1990. Porém, a eletroencefalografia ainda que tenha surgido há muito tempo, só passou a desempenhar um papel fundamental nos estudos de processos cerebrais complexos nos últimos anos, com o desenvolvimento de dispositivos eletrônicos e técnicas de processamento de sinais eficientes ${ }^{(11)}$.

A observação comportamental também foi uma medida de resposta muito usada, 5 dos 10 estudos usaram esse tipo de medida. No entanto, as outras utilizadas, como observações clínicas, observação da respiração e pulsação, medidas fisiológicas, medidas de pressão intracraniana e pressão de perfusão cerebral, também mostraram resultados satisfatórios.

Em relação aos propósitos dos estudos, procedimentos e principais resultados, os trabalhos foram descritos individualmente.

Foi realizado um estudo com 1 paciente, publicado em 1971, com o objetivo de comparar EEG normal com EEG de um paciente não-responsivo (coma vegetativo) somado a alguns estímulos ${ }^{(12)}$.

Estímulos luminosos ("Flashes") e sonoros ("clicks") foram distribuídos em intervalos não periódicos de 1 a 2 segundos. O estudo incluiu observações clínicas, EEG diurnos e noturnos, potenciais sensoriais evocados e correlações patológicas.

O principal resultado foi que o paciente não-responsivo apresentou padrões no EEG que se parecem com o de estado de vigília, embora o comportamento de alerta não tenha sido observado. Respostas evocadas para os "clicks" e a fase de movimento rapido ocular (REM) do sono foram ausentes.

Uma pesquisa realizada ${ }^{(13)}$,cuja amostra compreendeu 3 pacientes, publicada em 1983, teve o propósito de verificar como a produção de comportamentos através de estímulos pode ser usada para avaliar o controleambiental das respostas evidentes dos pacientes comatosos em estado vegetativo.

O experimento seguia os seguintes procedimentos: (1) O nome do paciente era falado; (2) um pedido verbal era feito para o paciente mover seus dedos, sua boca ou seus olhos; (3) a resposta tinha que ocorrer dentro de 10 segundos após o pedido verbal para ser pontuada; (4) se o paciente tivesse ausência de comportamentos ocorria 15 segundos de silêncio; se o paciente tivesse os comportamentos almejados tocava-se 15 segundos a sua música preferida, imediatamente depois. Cada sessão conteve 33 testes, 11 testes para cada um dos três comportamentos.

Os resultados mostraram que a música como recompensa afetou todos os três comportamentos para o paciente I, mas foi menos efetivo para os dois dos três comportamentos nos pacientes II e III que estavam em coma por um período mais extenso.

Foi publicado em $1983^{(14)}$, um estudo com 100 pacientes com o objetivo de comparar os resultados da reação vestibular calórica (CVR) e das respostas auditivas na base cerebral (ABR) em pacientes comatosos; verificar qual teste representa mais precisamente a atividade base cerebral dos pacientes em coma profundo; e que teste é capaz de fazer um prognóstico mais exato.

O ABR foi gravado no centro da testa do paciente, próximo a linha capilar e no lobo do orelha. Cada teste consistiu de 2,048 estímulos click e pelo menos dois testes foram feitos em cada orelha de cada paciente. A latência das ondas foi medida. A média de testes em cada paciente foi 5,2 vezes. A CVR foi feita por $20 \mathrm{ml}$ de solução fria $\left(4^{\circ} \mathrm{C}\right)$ injetada dentro do meato auditivo externo durante um período de 10s. Durante a irrigação, o ouvido foi elevado a $30^{\circ}$ e os olhos do paciente foram observados.

A latência inter-onda da onda I para a V (teste ABR) foi o principal resultado desse estudo, desde então essa latência inter-onda representa o tempo de condução dos sinais auditivos na base cerebral. Na primeira avaliação da ABR, 54 pacientes mostraram normalidade e 46 ABRs anormais. 26 dos $30(87 \%)$ pacientes que recuperam mostraram ABRs normais. Se total ou parcial desaparecimento (onda I) do ABR é observado em pacientes comatosos, pode-se prever um prognóstico ruim ou morte. Apenas 7 dos 26 que se recuperaram exibiram CVRs normais na primeira avaliação. $O$ teste $A B R$ representa mais precisamente a atividade base cerebral dos pacientes em coma profundo e mostrou-se mais eficaz em relação ao prognóstico.

Em 1990, foi publicado um relato de experiência contando o caso de um paciente que sofreu um dano cerebral severo, devido a uma agressão ${ }^{(15)}$. O paciente, um ano após o acidente, mantinha-se num "persistente estado de coma vegetativo".

Primeiramente, foi perguntado a um familiar o tipo de gosto musical do paciente. As músicas foram escolhidas e eram colocadas para o paciente ouvir. Através da música, conseguiu-se uma reação comportamental do paciente que comprovasse a existência de audição, bem como uma possibilidade de comunicação com ele. Em uma das vezes, a esposa trouxe uma fita com músicas que ela havia selecionado. O paciente ficou muito parado, aparentemente ouvindo a fita com atenção. De repente, uma ruga de expressão começou a aparecer em sua face, seus olhos se tornaram escuros e ele balançou vigorosamente a cabeça deslocando os fones de ouvido. Em um dos seus sons 
favoritos, a esposa encontrou o que ela gostaria de dizer a ele. A música falava da dor de perder alguém que você ama e também tinha uma promessa de nunca esquecer e nunca parar de amar, mesmo com a separação.

Em outro estudo publicado em 1990, foi elaborada uma nova forma de musicoterapia testada em 5 pacientes ${ }^{(6)}$. Esta consistia em uma canção improvisada e sem palavras, vocalizada por uma terapeuta, que acompanhava a pulsação e o ritmo respiratório do paciente.

Cada contato de musicoterapia durou, no máximo, entre 8 e 12 minutos. A característica da respiração do paciente determinava a essência da canção. A canção era expressada claramente, tanto que, quando vista qualquer reação do paciente, aquela parte da canção poderia ser repetida. A equipe da unidade foi orientada para estar em silêncio durante esse período e não fazer procedimentos invasivos no paciente por 10 minutos após o contato.

Existiu uma variedade de reações: mudança na respiração (ela se tornava mais lenta e mais profunda), movimentos do tipo motor fino, movimentos de fechar as mãos e virar a cabeça, abrir os olhos para a recuperação da consciência. Quando a terapeuta começava a cantar havia uma diminuição da pressão arterial. Então a pressão arterial se elevava rapidamente e se sustentava nesse nível elevado até o final do contato. Isto pode indicar uma tentativa de orientação e processo cognitivo dentro do contexto comunicacional. Medidas de EEG mostraram uma desincronização do ritmo theta, para o ritmo alfa ou ritmo beta em áreas de padrão sincronizado. Esse efeito, indicando excitação e atividade perceptiva, enfraqueceu-se depois que a musicoterapia parou. Um outro benefício desse estudo com musicoterapia foi que a equipe ficou ciente da qualidade e intensidade do contato humano.

Um estudo realizado em 5 pacientes, publicado em 1990, teve como objetivo determinar o efeito da entrada sensorial específica na atividade cortical de pacientes em estado de coma ${ }^{(16)}$.

Todos os dados foram coletados no mesmo período do dia, entre 18 e 21 hs, quando não há visitas e a unidade está relativamente silenciosa. Primeiramente, o paciente era avaliado segundo a Escala de Coma de Glasgow; após a colocação do fone de ouvido ele não era perturbado por alguns minutos, para garantir que qualquer resposta fosse apenas do estímulo auditivo; a gravação do EEG foi feita por 5 minutos: 1 min antes do estímulo musical, 3 min durante a música e 1 min depois do fim da música. Cada paciente foi observado 8 vezes. No $1^{\circ}$ dia foi gravado um eletro sem estímulo e em seguida com a anedota. Do $2^{\circ}$ ao $6^{\circ}$ dia foi colocado o estímulo musical. No $7^{\circ}$ dia foi anotada a pontuação da Escala de Glasgow, mas nenhum estímulo foi feito. A última observação foi feita uma semana mais tarde.

Os resultados indicam que aquelas pessoas em coma podem responder ao estímulo auditivo. As respostas foram variadas nesta pequena amostra de 5 pacientes, mas algum tipo de resposta ocorreu. Dois pacientes demonstraram uma resposta no EEG e os outros três responderam pela abertura ocular ou movimentação de extremidade.

Em estudo realizado com 1 paciente em 1994 $4^{(17)}$, o objetivo foi determinar se pacientes comatosos respondem diferencialmente a 4 tipos de estímulos auditivos e determinar que medidas fisiológicas e observações comportamentais melhor captam as mudanças na responsividade.

Os dados foram coletados num período de 14 dias consecutivos ( 2 sessões por dia de 20 minutos). Apenas 1 dos 4 tipos de estímulo foi apresentado durante cada sessão em uma seqüência randomizada, 7 vezes cada estímulo. As observações comportamentais foram filmadas e analisadas por 2 alunos de graduação que não foram informados da proposta do estudo. A mensuração das medidas fisiológicas foi realizada num intervalo de 15 segundos para o pulso, 30 segundos para a respiração e 30 segundos para os comportamentos.

Em relação aos resultados, o estímulo de vozes familiares e amigos foi o que produziu um maior aumento na taxa de pulsação em relação a taxa basal e este foi durante os primeiros 90 segundos, bem como o que resultou no maior número de movimentos corporais. Quanto aos outros 3 estímulos, a música clássica e sons da natureza se mantiveram próximos a linha basal para a pulsação e respiração, ficando o rock'roll (estilo de preferência) predominantemente abaixo da linha basal. Os pacientes comatosos podem responder diferencialmente a vários tipos de estímulos auditivos. Movimentos corporais e medidas da taxa de pulsação melhor evidenciaram a responsividade do paciente.

Com o propósito de avaliar os efeitos de estímulos auditivos na pressão intracraniana (ICP) e pressão de perfusão cerebral (CPP) em pacientes com traumatismo cerebral severo, foi realizado um estudo com 15 pacientes em $1995^{(18)}$. Para isso, cada sujeito foi testado 2 vezes: a primeira entre 36 e $48 \mathrm{hs}$ de admissão e a segunda entre 84 e $96 \mathrm{hs} \mathrm{da}$ admissão. Cinco minutos de ICP foram gravados antes da manipulação de qualquer estímulo. Cada intervenção durou 15 minutos. Os três tipos de estímulos foram aplicados em ordens randomizadas. As medidas de ICP e CPP foram gravadas a cada 30 segundos através de um computador. Houve um período de 10 minutos entre cada manipulação para permitir o retorno do ICP e CPP a medida basal. Durante os 15 minutos de intervenção, houve um grande empenho para assegurar que nenhuma outra manipulação estava ocorrendo simultaneamente.

Os resultados não mostraram mudanças estatísticas significantes, nem clinicamente consideráveis, na pressão intracraniana e na pressão de perfusão cerebral durante o período estudado. A literatura disponível sobre o efeito da estimulação auditiva em pacientes com pressão intracraniana é contraditória e inconclusiva. 
Alguns autores investigaram o "efeito Mozart" na atividade epiliforme no EEG em 29 pacientes $^{(19)}$. EEGs foram gravados utilizando o padrão internacional de localização de eletrodos. O número de descargas focais ou duração das manifestações generalizadas foram contadas. No caso de estados epilépticos, a duração dos eventos foi mensurada durante um certo período de tempo. O número de descargas e durações das manifestações foram contadas durante 5 estados diferentes (cada um usualmente por 10 minutos): (1) antes; (2) durante a música de Mozart; (3) depois; (4) durante a música controle (Old Time Pop Piano Tunes) e (5) depois de tudo.

Os principais resultados foram que em 23 de 29 ocasiões $(79 \%)$, diminuições significativas na atividade epiliforme foram notadas em pacientes mesmo em estado de coma, com estado epilético ou descarga epiliforme lateralizado periódico (PLEDs). A mudança na quantidade da atividade epiliforme em um paciente em coma era de $62 \%$ antes da música e $21 \%$ durante Mozart. As amplitudes dessas descargas também diminuíram freqüentemente. Mapeamento cerebral durante a música mostrou diminuição das atividades alfa e teta nas áreas centrais, enquanto aumentou as ondas delta na área mediana frontal. É provável que a superorganização do córtex cerebral com suas colunas radiais altamente estruturadas vistas em ambos os hemisférios possa ressonar com a arquitetura superior da música de Mozart.

Foi elaborado um estudo com o propósito de descrever as respostas de sujeitos normais para a modulação de altura e timbre de sons que se parecem com os tons que ocorrem na música, e apresentar resultados preliminares em 2 pacientes comatosos ${ }^{(20)}$.

Os estímulos foram tons instrumentais produzidos por um gerador e controlados por um computador. $\mathrm{O}$ estímulo foi apresentado em ambos os ouvidos em $45 \mathrm{~dB}$ SL e outro canal estéreo do programa sonoro foi usado para verificar a média. Os sujeitos do grupo controle estavam todos acordados mas não instruídos para atender

\section{REFERÊNCIAS}

1. Lusvarghi L. O cérebro deixa de ser um mistério. Medicis 2000; 4:18-24.

2. James W. Principles of psychology [monografy on the Internet]. New York; 1890. [ cited 2004 Oct 25]. Available from: http://psychclassics.yorku.ca/James/Principles/

3. Campos A, Santos AMG, Xavier GF. A consciência como fruto da evolução e do funcionamento do sistema nervoso. Psicol USP. 1997;8(2):181-226.

4. Rabello GD. Coma e estados alterados de consciência. In: Nitrini R, Bacheschi LA. A neurologia que todo médico deve saber. São Paulo: Atheneu; 2003. p. 143-69.

5. Silva AL, Schlicknann CG, Faria JG. O coma e seu impacto no processo de ser e viver: implicações para o cuidado de enfermagem. Rev Gaúcha Enferm. 2002;23(2):81-107.

6. Aldridge D, Gustorff D, Hannich HJ. Where am I ? Music ao estímulo. Eletrodos gravaram potenciais evocados.

Os resultados preliminares nesses 2 pacientes não-responsivos sugerem uma larga variação no funcionamento cortical, pois um paciente em coma apresentou potenciais evocados essencialmente normais e o outro em estado vegetativo persistente não apresentou potenciais corticais identificáveis. Certamente há uma grande distância a ser percorrida antes de ser estabelecido qual deve ser a tendência geral e quais serão as implicações para o cuidado desse tipo de paciente. Foi sugerido, entretanto, que resultados desse tipo podem alterar a visão sobre o estado comatoso ou os diferentes pacientes não responsivos.

Todos os estudos mencionados convergem para uma tentativa de avaliar e validar a audição dos pacientes em estado de coma. E essa preocupação trás, de modo implícito, uma vontade de humanização na assistência direta com esse tipo de paciente.

\section{CONCLUSÕES}

A maior parte dos estudos selecionados foi publicada no período de 1971-1995 (80\%), pelos Estados Unidos $(70 \%)$, como pesquisa longitudinal $(50 \%)$ ou relato de caso (30\%); a amostra em geral variou entre 1 e $5(70 \%)$, a maioria usou a música (80\%); o EEG $(60 \%)$ e a observação comportamental $(50 \%)$ foram as medidas de respostaao estímulo mais utilizadas. Os procedimentos foram muito variados, porém a maioria dos estudos, de uma maneira ou de outra, apontam para a existência de uma percepção auditiva nos pacientes em estado de coma.

Mais estudos precisam ser feitos para determinar outros métodos capazes de medir as respostas de pacientes comatosos a um estímulo auditivo, pois o universo de estudos nessa linha ainda é muito pequeno e incerto. A neurociência tem avançado muito, mas para muitos assuntos, como o nosso, ainda não há consenso. O paciente em coma é um grande questionamento...

therapy applied to coma patients. J R Soc Med. 1990;83(6): 345-6.

7. Kandel ER, Schwartz JH, Jessell TM, editors. Fundamentos da neurociência e do comportamento. Rio de Janeiro: Guanabara Koogan; 1997.

8. Ratey JJ. O cérebro: um guia para o usuário: como aumentar a saúde, agilidade e longevidade de nossos cérebros através das mais recentes descobertas cientificas. Rio de Janeiro: Objetiva; 2002.

9. Silva MJP, Dobbro ELR. Reflexões sobre a importância da mente na recuperação do paciente em coma. Mundo Saúde. 2000; 24(24):249-54.

10. Johns U. Do silêncio à fala: estudo de caso de uma menina com mutismo seletivo. In: Ruud E, organizador. Música e saúde. São Paulo: Summus; 1991. p. 15-8. 
11. Araújo DB, Santos AC, Sakamoto A, Baffa O. Imagens do cérebro em ação. Ciênc Hoje. 2003;33(197):28-35.

12. Wilkus RJ, Harvey F, Ojemann LM, Lettich E. Electroencephalogram and sensory evoked potentials. Findings in an unresponsive patient with pontine infarct. Arch Neurol. 1971; 24(6):538-44.

13. Boyle ME, Greer RD. Operant produces and the comatose patient. J Appl Behav Anal. 1983;16(1):3-12.

14. Yagi T, Baba S. Evaluation of the brain-stem function by the auditory brain-stem response and the caloric vestibular reaction in comatose patient. Arch Otorhinolaryngol. 1983; 23(8):33-43

15. Jones CP. Spark of life. Geriatr Nurs 1990; 11(4):194-6.

16. Sisson R. Effects of auditory stimuli on comatose patients with head injury. Heart Lung. 1990;19(4):373-8.

17. Jones R, Hux K, Morton-Anderson AK, Knepper L. Auditory stimulation effect on a comatose survivor of traumatic brain injury. Arch Phys Med Rehabil. 1994;75(2):164-71.

18. Schinner KM, Chisholm AH, Grap MJ, Siva P, Hallinan M, La Voice-Hawkins AM. Effects of auditry stimuli on intracranial pressure and cerebral perfusion pressure in traumatic brain injury. J Neurosci Nurs. 1995;27(6):348-54.

19. Hughes JR, Daaboul Y, Fino JJ, Shaw GL. The "Mozart effect" on epileform activity. Clin Electroenceephalogr. 1998; 29(3):109-19.

20. Jones SJ, Pato MV, Longe O. Auditory information processing in comatose patients: Eps to synthesized "musical" tones. Electroencephalogr Clin Neurophysiol Suppl. 1999; 50:402-7. 